\title{
Development of an Analytical Method to Predict the Behaviour of the Magnetic Field in PM Linear Motors with Halbach Array
}

\author{
Marilia A. da Silveira, Leandro D. Marques, \\ Lutheran University of Brazil-ULBRA, Av. Farroupilha, 8001, Canoas-RS, Brazil, marilia.amaral@ulbra.br, \\ leandro.marques@aes.com \\ Aly F. Flores Filho, Felipe Treviso \\ Federal University of Rio Grande do Sul-UFRGS, Av. Osvaldo Aranha, 103, Porto Alegre-RS, Brazil, \\ aly.flores@ufrgs.br,felipe.treviso@ufrgs.br
}

\begin{abstract}
This paper describes the application of the method of separation of variables and the use of Fourier series for solving the Laplace's and Poisson's equations on the study and analysis of the magnetic field produced in a linear motor with Halbach array. Equations for predicting the 2D magnetic flux density distribution produced in the air gap were developed. The model was validated by means of finite element analysis and by measurements carried on a prototype of the linear motor. Theoretical results helped understand the behaviour of the magnetic flux density in the air gap and to obtain the values of the static propulsion force and normal force in such machine.
\end{abstract}

Index Terms - Analytical model, Fourier series, Halbach array, Laplace's and Poisson's equations, PM linear motor.

\section{INTRODUCTION}

Fourier series can be applied on the solution of many problems in Electrical Engineering, especially on the study of electrical circuits and on the electric and magnetic field analysis. In the magnetic field analysis, it can be employed for predicting the magnetic flux density distribution in electromagnetic devices and for calculating the involved forces. The Fourier series was presented by Jean Baptiste Joseph Fourier (1768-1830) in his Théorie Analytique de la Chaleur, in 1822 [1]. It allows the representation of a periodic nonsinusoidal function in terms of an infinite sum of sines and cosines. Maxwell's equations form a set of partial differential equations that can describe the behaviour of magnetic and electric fields and the relations between them. In order to obtain the equations of fields in the electric motors, the solution of Laplace's and Poisson's equations can be involved. These equations are subject of study in Electrical Engineering courses, principally due to their application on electromagnetic problems.

On the study of the magnetic field in electromagnetic linear motors, the equation of the magnetic flux density demands the representation of the device under study in a model by using a suited system of co-ordinates, where boundary conditions are imposed. The model is based on the magnetic circuit of the device and must be divided into regions, and the electric and magnetic properties of those regions must be defined. The conditions of the magnetic field between the regions are specified, too. 
The analysis can be done by means of the magnetic vector potential or, for regions free of current, in terms of the magnetic scalar potential formulation. The method of separation of variables for solving the Laplace's and Poisson's equations can be applied too, and the solution leads to the Fourier series for devices represented in rectangular coordinates. In cylindrical devices, such as tubular linear electromagnetic actuators analysed by the use of cylindrical coordinates, the solution is represented in terms of Fourier series and Bessel functions [2] [3].

Electromagnetic linear motors can rely on a translator and a stator. In such devices, the translator can develop movement through a straight line directly by means of a linear propulsion force produced by electromagnetic interaction between the translator and the stator. This type of motor does not require mechanical systems that convert rotation into linear movement. It is suitable for applications that require development of propulsion force in a specific direction.

In graduate and undergraduate courses, the study and development of electromagnetic linear devices is one of the lines of work in Electrical Engineering. In such undergraduate courses, the study of the Laplace's and Poisson's equations for the solution of the electric or magnetic field is present on Electromagnetism syllabus. Commonly, the bibliography suggests exercises where the application of those equations is used for the solution of the electric potential in problems related to the electric field content.

A proposed case of study about the use of Fourier series, Laplace's and Poisson's equations applied to an electromagnetic device is a simple electromagnetic linear motor that can rely on a mover with permanent magnets and a stator with armature windings mounted around a slotless ferromagnetic armature core. In such devices, the mover can develop movement through a straight line by means of a propulsion force. The focus of an analytical study is to obtain the equations of the magnetic field and the involved forces.

In this paper, the equations of the magnetic field and the propulsion and normal forces produced by a device with permanent magnets arranged in a Halbach array are deduced. In the device, a nonferromagnetic plate holds the permanent magnets, because in that kind of array the magnetic field is established through a path that involves only one polar surface of the permanent magnets. As part of the study, a prototype of the device was built in the laboratory and was employed for validation purposes of the analytical model.

\section{STUDY OF A DC LINEAR MOTOR WITH PERMANENT MAGNETS IN A QUASI-HALBACH ARRAY}

The analytical model presented in this paper was deduced for a DC linear motor as shown by Fig. 1. It has a mover or translator with permanent magnets in a quasi-Halbach array topology and a stator with armature winding mounted around a slotless ferromagnetic armature core [9]. Table I presents the principal characteristics of the linear motor constructed. It has nine $\mathrm{NdFeB}$ permanent magnets arranged in a quasi-Halbach array assembled on an aluminium plate. The armature core is made of 1020 steel with resistivity of $0.1862 \mu \Omega \mathrm{m}$, and maximum relative permeability is 1,400 . In such 
devices, the mover can develop movement through a straight line by means of a linear propulsion force. The array was mounted in order to have the magnetic flux established through a path that involves only one polar surface of the permanent magnets, the air gap and the ferromagnetic armature core. This means that the magnetic flux that passes through the upper polar surface of array core is very weak, and a nonmagnetic core can be used only to support the permanent magnets mechanically.

The mass reduction due to the employment of a nonmagnetic material in the PM assembly is desirable when high acceleration is required. The magnetic flux density distribution in the air gap presents a sinusoidal shape in a Halbach array instead a flattened sinusoidal shape produced in the PM linear motors without this kind of array. In the case of a flattened sine shape, the wave becomes squarer, with many odd number harmonics. That results in electromagnetic force ripple and variable force.

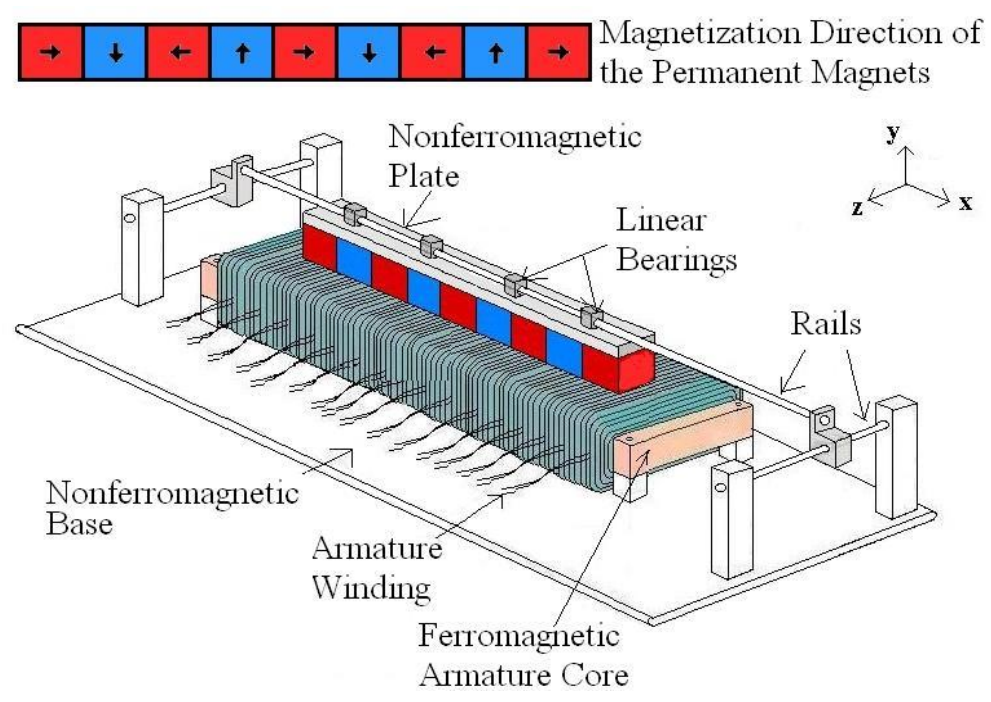

Fig. 1. Linear motor under study.

TABLE I. PRINCIPAL CHARACTERISTICS OF THE LINEAR MOTOR WITH HALBACH ARRAY

\begin{tabular}{lc}
\hline \multicolumn{1}{c}{ Item } & Feature \\
\hline Number of phases (coils) of the armature winding & 12 \\
Turns per phase & 150 \\
Armature core material & 1020 Steel \\
PM number & 9 \\
PM dimensions & $10(\mathrm{~B}) \times 10(\mathrm{~W}) \times 10(\mathrm{~T})[\mathrm{mm}]$ \\
PM material & Sintered anisotropic $\mathrm{NdFeB}$ \\
Air gap & $12(\mathrm{~L})[\mathrm{mm}]$ \\
\hline
\end{tabular}

\section{A. Analytical Model of the Magnetic Field Produced by Permanent Magnets}

The aim of the development of an analytical model was to obtain the equations of the magnetic field and the involved forces produced by the DC linear motor. The method was based on an analysis of a 2D model in rectangular coordinates. The magnetic circuit of the linear motor is divided into regions through the $y$-axis, and boundaries denoted by $O, B, G$ and $P$, Fig. 2, The nonmagnetic plate was omitted in the model because the magnetic flux was concentrated between the permanent magnets and 
the armature core. Only the coil phases located under the permanent magnets with magnetization through the y-axis are responsible for the production of linear propulsion force. Four permanent magnets were represented in the model, according to Fig. 2, where $l_{g}$ is the air gap length, $l_{m}$ is the permanent magnets axial length, $l_{b}$ is the thickness of the coils, and $l_{t}$ corresponds to one side of the area considered for the analysis, normal to the $y$-component of the magnetic flux density vector, with $l_{t} / 4$ corresponding to the side of the square polar area of each permanent magnet.

The distribution of the magnetic flux density established in the air gap depends on the magnetic field produced by the permanent magnets and on the magnetic field produced by the currents in the phases of the armature windings. The magnetic field due to the permanent magnets was analysed separately from the magnetic field produced by the armature windings. So, the magnetic field due to the current in the windings is not considered by the present analysis and the region between the boundaries $O$ and $B$ has the same magnetic properties as the air.

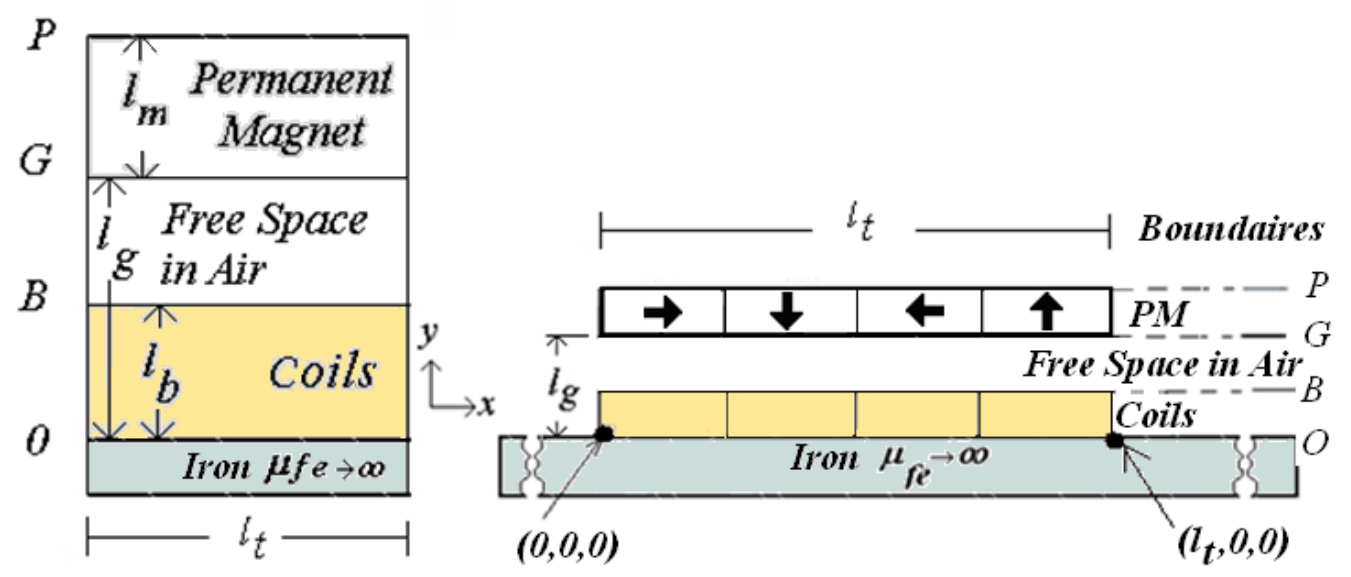

Fig. 2. Model of the motor under study, with boundaries and regions.

In the free space of air, the magnetic flux density vector, $\vec{B}$, and the magnetic field vector, $\vec{H}$, are related by $\vec{B}=\mu_{0} \vec{H}$, where $\mu_{0}$ is the magnetic permeability of the vacuum. The analytical model was developed in terms of the magnetic scalar potential, $\psi$ [5]. So, the magnetic field, $\vec{H}$, is equal to the negative gradient of $\psi$, according to $\vec{H}=-\nabla \psi$. Applying this equation to the second Maxwell's equation, $\nabla \cdot \vec{B}=0$, and taking into account the relation between $\vec{B}$ and $\vec{H}$, one can obtain the Laplace's equation in terms of the magnetic scalar potential for the air gap, $\psi_{g}$, given by $\nabla^{2} \psi_{g}=0$. In $2 \mathrm{D}$ rectangular coordinates, it can result in:

$$
\frac{\partial^{2} \psi_{g}}{\partial x^{2}}+\frac{\partial^{2} \psi_{g}}{\partial y^{2}}=0
$$

A solution method to (1) involves the determination of a field function, which satisfies the Laplace's equation, the imposed boundaries and the field conditions in the permanent magnets region [2]-[4]. The method of separation of variables allows solving (1). Taking into account that $\psi_{g}$ can be 
expressed as the product of two functions, $X(x)$, and $Y(y)$, (1) is modified to $\psi_{g}(x, y)=X(x) Y(y)$ [4] [5], where $X(x)$ is a function that depends only on $x$, and $Y(y)$ depends only on $y$. After some operations, one can get (2).

$$
\frac{1}{X} \frac{d^{2} X}{d x^{2}}+\frac{1}{Y} \frac{d^{2} Y}{d y^{2}}=0
$$

The sum of the terms in the left side of (2) is equal to zero, and the variables are independent, so, each term is equal to a constant and the variables can be separated according the set of two equations presented in (3) [5] [6]:

$$
\frac{1}{X} \frac{d^{2} X}{d x^{2}}=\alpha^{2} \text { and } \quad \frac{1}{Y} \frac{d^{2} Y}{d y^{2}}=\beta^{2}
$$

and $\alpha^{2}+\beta^{2}=0$. If $\beta$ is a real quantity, $\alpha$ must be an imaginary and $\beta=\sqrt{-\alpha^{2}}$. The solution to (3) is given by $X(x)=A_{1} e^{\alpha x}+B_{1} e^{-\alpha x}$ and $Y(y)=A_{2} e^{\beta y}+B_{2} e^{-\beta y}$. When these solutions are applied in the magnetic scalar potential equation, it results in:

$$
\psi_{g}(x, y)=\left(A_{1} e^{\alpha x}+B_{1} e^{-\alpha x}\right)\left(A_{2} e^{\beta y}+B_{2} e^{-\beta y}\right)
$$

The magnetic scalar potential was considered equal to zero on the planes $x=0, x=l_{t} / 2, x=l_{t}, y=0$ and $y=l_{g}+l_{m}$. By means of the conditions $\psi_{g}=0$ on $x=0$, one can get $B_{1}=-A_{1}$, and on $x=l_{t} / 2$ leads to $X\left(l_{t} / 2\right)=A_{1}\left(e^{\alpha\left(l_{t} / 2\right)}-e^{-\alpha\left(l_{t} / 2\right)}\right)=0$, or $e^{2 \alpha\left(l_{t} / 2\right)}=1$. Applying Euler identity, one can get $\alpha=j \frac{2 \pi n}{l_{t}}$ and $\beta=\frac{2 \pi \mathrm{n}}{l_{t}}$. By these results, $X(x)$ can be represented as $X(x)=j 2 A_{1} \sin \left(\frac{2 \pi \mathrm{n}}{l_{t}} x\right)$ and $\psi_{g}$ can be represented by means of a Fourier series, according (5):

$$
\psi_{g}(x, y)=\sum_{n=1,3, \ldots}^{\infty}\left(j 2 A_{1} \sin (\kappa n x)\right)\left(A_{2} e^{\kappa n y}+B_{2} e^{-\kappa n y}\right)=\sum_{n=1,3, \ldots}^{\infty}\left(k_{1} e^{\kappa n y}+k_{2} e^{-\kappa n y}\right) \sin (\kappa n x)
$$

where $n$ is an integer and $\kappa$ is defined by $\kappa=2 \pi / l_{t}$.

In the ferromagnetic materials, $\vec{B}$ is obtained by $\vec{B}=\mu_{o}(\vec{M}+\vec{H})$, where $\vec{M}$ represents the magnetization vector. Again, applying $\vec{H}=-\nabla \psi$ to the second Maxwell's equation, $\nabla \cdot \bar{B}=0$, and taking into account the relation between $\vec{B}$ and $\vec{M}$, one can obtain the Poisson equation in terms of the magnetic scalar potential for the permanent magnets region, $\nabla \psi_{p m}$ [8]. It results in $\nabla^{2} \psi_{p m}=\nabla \vec{M}$. The magnetic scalar potential in the PM region is obtained by $\psi_{p m}=\psi_{h}+\psi_{p}$, where $\psi_{h}$ is a homogeneous term and must satisfy the Laplace's equation $\nabla^{2} \psi_{h}=0$, and $\psi_{p}$ is the particular term, solved by Poisson's equation $\nabla^{2} \psi_{p}=\nabla \vec{M}$ [2]-[7]. The magnetization vector, $\vec{M}$, 
with components through the $x$ and $y$-axis, is represented by $M_{x} \vec{i}+M_{y} \vec{j}$. Each component can be represented by a Fourier series. Fig. 3 shows the graphs of the components of the magnetization vector. The Fourier series that represents the behaviour of the $x$-component of the magnetization vector is presented in (6). The $y$-component is represented by (7).

$$
\begin{aligned}
& M_{x}=\frac{M_{O}}{\pi} \sum_{n=1,3, . .}^{\infty} \frac{1}{n}\left[\left(\left(\sin \left(\frac{\pi n}{2}\right)-\sin \left(\frac{3 \pi n}{2}\right)\right) \cos (\kappa n x)\right)+((1-\cos (\pi n)) \sin (\kappa n x))\right] \\
& M_{y}=\frac{M_{O}}{\pi} \sum_{n=1,3, . .}^{\infty} \frac{1}{n}\left[\left(\left(\sin \left(\frac{\pi n}{2}\right)-\sin \left(\frac{3 \pi n}{2}\right)\right) \cos (\kappa n x)\right)+((\cos (\pi n)-1) \sin (\kappa n x))\right]
\end{aligned}
$$

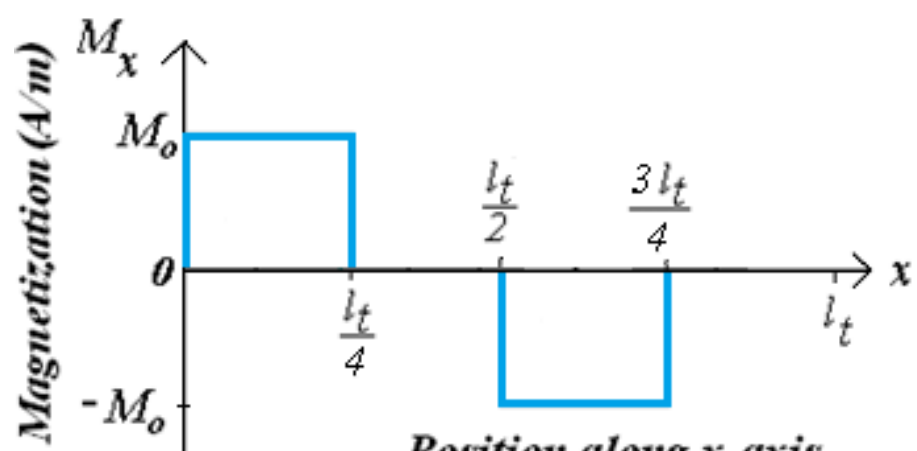

(a)

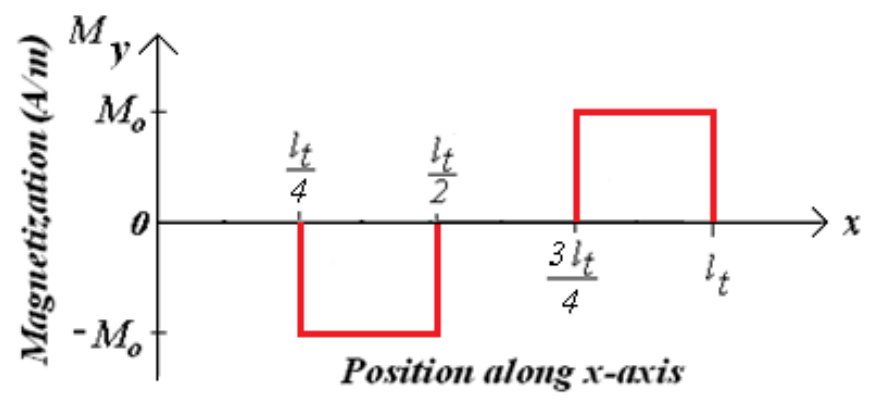

(b)

Fig. 3. Graphs of the components of the magnetization vector: (a) $x$-component and (b) $y$-component.

The homogeneous term of $\psi_{\mathrm{pm}}$ is calculated following the same steps employed to obtain (5) and it is equal to (8).

$$
\psi_{h}=\sum_{n=1,3, \ldots}^{\infty}\left(k_{3} e^{\kappa n y}+k_{4} e^{-\kappa n y}\right) \sin (\kappa n x)
$$

The particular term, $\psi_{p}$, is solved by Poisson's equation $\nabla^{2} \psi_{p}=\nabla \cdot \vec{M}$, presented in (9).

$$
\nabla^{2} \psi_{p}=\frac{\kappa M_{O}}{\pi} \sum_{n=1,3, .}^{\infty}\left[((1-\cos (\pi n)) \cos (\kappa n x))-\left(\left(\sin \left(\frac{\pi n}{2}\right)-\sin \left(\frac{3 \pi n}{2}\right)\right) \sin (\kappa n x)\right)\right]
$$


whose simplest solution is obtained by integrating twice the right side of (9) through $x$. It results in:

$$
\psi_{p}=\frac{M_{O}}{\kappa \pi} \sum_{n=1,3, .}^{\infty} \frac{1}{n^{2}}\left[((\cos (\pi n)-1) \cos (\kappa n x))+\left(\left(\sin \left(\frac{\pi n}{2}\right)-\sin \left(\frac{3 \pi n}{2}\right)\right) \sin (\kappa n x)\right)\right]
$$

The equation of the magnetic scalar potential in the PM region, $\psi_{p m}$, is defined by (11):

$$
\psi_{p m}(x, y)=\sum_{n=1,3, \ldots}^{\infty}\left(\left(k_{3} e^{\kappa n y}+k_{4} e^{-\kappa n y}\right) \sin (\kappa n x)\right)+\psi_{p}
$$

Constants $k_{1}, k_{2}, k_{3}$ and $k_{4}$ are obtained by means of the boundary conditions. The boundary conditions are the following:

- the magnetic scalar potential in the air gap on boundary $O$, Fig. 2, where $y=0, \psi_{g}^{O}$, is equal to zero and this results in $k_{2}=-k_{1}$;

- the magnetic scalar potential in the PM region on the boundary $P$, Fig. 2, where $y=\left(l_{g}+l_{m}\right), \psi_{p m}^{P}$, is equal to zero and this results in $k_{4}=-k_{3} e^{2 \beta\left(l_{m}+l_{g}\right)}-\psi_{p} \frac{e^{\beta\left(l_{m}+l_{g}\right)}}{2 \sin (\kappa n x)} ;$

- on boundary $G$, Fig. 2, where $y=l_{g}$, the $y$-component of the magnetic field in the air gap, $H_{g_{y}}^{G}$ and in the permanent magnets, $H_{p m_{y}}^{G}$ are related by $H_{g_{y}}^{G}=H_{p m_{y}}^{G}+M_{y}$, and the respective magnetic scalar potentials are related by $\psi_{\mathrm{g}}^{G}=\underset{\mathrm{pm}}{G}$.

Those conditions allow one to obtain the equations of the potentials. Eq. (12) presents the expressions of the magnetic scalar potential in the air gap:

$$
\begin{aligned}
\psi_{g}(x, y) & =\sum_{n=1,3, .}^{\infty}\left(\frac{\left(e^{k n y}-e^{-k n y}\right)}{2\left(e^{k n l_{g}}-e^{-k n l_{g}}\right)\left(e^{2 \kappa n\left(l_{m}+l_{g}\right)}-1\right)}\right) \\
& {\left[\begin{array}{l}
{\left[\begin{array}{l}
\psi_{p}\left(\left(e^{k n l_{g}}-e^{k n\left(2 l_{m}+l_{g}\right)}\right)\left(e^{k n l_{g}}+e^{-k n l_{g}}-2 e^{k n\left(l_{m}+l_{g}\right)}\right)\right. \\
\left.+2\left(e^{2 k n\left(l_{m}+l_{g}\right)}-1\right)\left(1-e^{k n l_{m}}\right)\right)
\end{array}\right]} \\
+\left[\left(\frac{M_{y}}{\kappa n}\right)\left(e^{k n l_{g}}-e^{-k n l_{g}}\right)\left(e^{k n l_{g}}-e^{k n\left(2 l_{m}+l_{g}\right)}\right)\right]
\end{array}\right] }
\end{aligned}
$$

The $x$-component of the magnetic flux density in the air-gap, $B_{g_{x}}$, obtained by $-\mu_{0}\left(\partial \psi_{g} / \partial x\right)$, resulting in: 


$$
\begin{aligned}
B_{g x} & =-\mu_{0} \kappa \sum_{n=1,3, .}^{\infty} n\left(\frac{\left(e^{k n y}-e^{-k n y}\right)}{2\left(e^{k n l_{g}}-e^{-k n l_{g}}\right)\left(e^{2 k n\left(l_{m}+l_{g}\right)}-1\right)}\right) \\
& {\left[\begin{array}{l}
{\left[\begin{array}{l}
\psi_{p}^{\prime}\left(\left(e^{k n l_{g}}-e^{k n\left(2 l_{m}+l_{g}\right)}\right)\left(e^{k n l_{g}}+e^{-k n l_{g}}-2 e^{k n\left(l_{m}+l_{g}\right)}\right)+\right. \\
\left.2\left(e^{2 k n\left(l_{m}+l_{g}\right)}-1\right)\left(1-e^{k n l_{m}}\right)\right)
\end{array}\right]} \\
+\left[\left(\frac{M_{y}^{\prime}}{\kappa n}\right)\left(e^{k n l_{g}}-e^{-k n l_{g}}\right)\left(e^{k n l_{g}}-e^{k n\left(2 l_{m}+l_{g}\right)}\right)\right.
\end{array}\right] }
\end{aligned}
$$

where $M_{y}^{\prime}$ and $\psi_{p}^{\prime}$ are given respectively by (14) and (15).

$$
\begin{gathered}
M_{y}^{\prime}=\frac{M_{o}}{\pi} \sum_{n=1,3, . .}^{\infty} \frac{1}{n}\left[\left(\left(\sin \left(\frac{3 \pi n}{2}\right)-\sin \left(\frac{\pi n}{2}\right)\right) \sin (\kappa n x)\right)+((\cos (\pi n)-1) \cos (\kappa n x))\right] \\
\psi_{p}^{\prime}=\frac{M_{o}}{\kappa \pi} \sum_{n=1,3, .}^{\infty} \frac{1}{n^{2}}\left[((1-\cos (\pi n)) \sin (\kappa n x))+\left(\left(\sin \left(\frac{\pi n}{2}\right)-\sin \left(\frac{3 \pi n}{2}\right)\right) \cos (\kappa n x)\right)\right]
\end{gathered}
$$

The $y$-component of the magnetic flux density in the air-gap, $B_{g_{y}}$, obtained by $-\mu_{0}\left(\partial \psi_{g} / \partial y\right)$, resulting in:

$$
\begin{aligned}
& B_{g_{y}}=-\mu_{0} \kappa \sum_{n=1,3, .}^{\infty} n\left(\frac{\left(e^{\kappa n y}+e^{-k n y}\right)}{2\left(e^{k n l_{g}}-e^{-k n l_{g}}\right)\left(e^{2 k n\left(l_{m}+l_{g}\right)}-1\right)}\right) \\
& {\left[\begin{array}{l}
{\left[\begin{array}{l}
\psi_{p}\left(\left(e^{\kappa n l_{g}}-e^{k n\left(2 l_{m}+l_{g}\right)}\right)\left(e^{k n l_{g}}+e^{-k n l_{g}}-2 e^{k n\left(l_{m}+l_{g}\right)}\right)+\right. \\
\left.2\left(e^{2 \kappa n\left(l_{m}+l_{g}\right)}-1\right)\left(1-e^{\kappa n l_{m}}\right)\right)
\end{array}\right]} \\
+\left[\left(\frac{M_{y}}{\kappa n}\right)\left(e^{k n l_{g}}-e^{-k n l_{g}}\right)\left(e^{k n l_{g}}-e^{\kappa n\left(2 l_{m}+l_{g}\right)}\right)\right.
\end{array}\right] }
\end{aligned}
$$

By using the expression of $B_{g_{y}}$, it was possible to obtain the equation of the propulsion force that acts over the mover of the linear motor. That force produces movement through the $\mathrm{x}$-axis and depends on the current density in the armature phases located under the permanent magnets and the $y$ component of the magnetic flux density in the air gap produced by those permanent magnets. The current density vector has only the $z$-component, i.e. $\vec{J}=J_{z} \vec{k}$, where $J_{z}$ is the $z$-component of the density current vector. In the region of the armature phases, the current density can be represented by a Fourier series, according (17):

$$
J_{z}=\frac{4 J_{o}}{\pi} \sum_{n=1,3, \ldots}^{\infty} \frac{\cos \left(\kappa n l_{d}\right)}{n} \sin \left(\kappa n\left(x-\frac{l_{t}}{8}\right)\right)
$$


where $l_{d}=\frac{l_{t}}{8}, J_{o}$ is the current density in each coil, given by $N I / s_{c}$, with $N$ equals to the number of turns of each coil, $I$ the current and $s_{c}$ the cross-sectional area of one coil defined by $s_{c}=l_{b} \frac{l_{t}}{4}$. Its behaviour if defined by the graph of Fig. 4 .

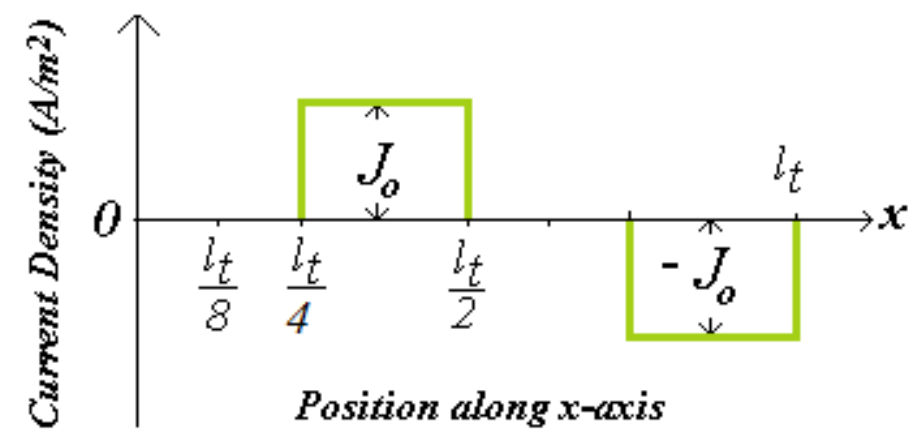

Fig. 4. Graph of the current density in the coils located under the permanent magnets.

\section{B. The Analytical Solution for the Magnetic Field Produced by the Armature Windings}

The magnetic field produced by the armature windings is obtained by means of the Poisson's equation expressed in terms of the magnetic vector potential, $\vec{A}$, related to the current density vector, $\vec{J}$. The effect of the magnetic field produced by the armature phases on the distribution of the resulting magnetic flux density is analysed separately from the effects of the magnetic field produced by the permanent magnets. Afterwards, the effect of both can be combined. By this way, in the model of the magnetic field produced by armature phases under current, the permanent magnets are represented only by a region located between the boundaries $G$ and the $P$, Fig. 2, where the relative magnetic permeability is equal to $\mu_{r_{p m}}$.

The calculation of the magnetic field in the region between boundaries $O$ and $B$, Fig. 2, is made by means of $\nabla \times \vec{H}=\vec{J}$. The vector $\vec{B}$ can be expressed as the curl of the magnetic vector potential, according $\vec{B}=\nabla \times \vec{A}$. For the linear motor under study, $\vec{J}=J_{z} \vec{k}$. Due to this, the magnetic vector potential, $\vec{A}$, whose generation is made by $J_{z}$, has only the z-component, $A_{b_{z}}$. Making the curl of $\vec{B}$, one can have $\nabla \times \vec{B}=\nabla \times \nabla \times \vec{A}=\mu_{O} \vec{J}$ [7]. After some operations, it is obtained a scalar equation that defined a relation between $J_{z}$ and $A_{b_{z}}$ by the Laplacian of the later, according to $\nabla^{2} A_{b_{z}}=-\mu_{o} J_{z}$. This is the Laplace's equation for the magnetic vector potential in the region of the armature phases and it can be expressed by (18):

$$
\nabla^{2} A_{b_{z}}=\frac{\partial^{2} A_{b_{z}}}{\partial x^{2}}+\frac{\partial^{2} A_{b_{z}}}{\partial y^{2}}=-\mu_{o} J_{z}
$$


The solution of (15) is equal to the sum of two terms, or $A_{b_{z}}=A_{b_{z p}}+A_{b_{z h}}$, where $A_{b_{z p}}$ is the particular term, solved by Poisson's equation, and $A_{b_{z} h}$ is the homogeneous term and must satisfy the Laplace's equation, $\nabla^{2} A_{b_{z h}}=0$ [7], or:

$$
\nabla^{2} A_{b_{z h}}=\frac{\partial^{2} A_{b_{z h}}}{\partial x^{2}}+\frac{\partial^{2} A_{b_{z h}}}{\partial y^{2}}=0
$$

In the solution of (19) is employed the same steps of the solution of (1) using the method of separation of variables. The magnetic vector potential is consider equal to zero on the planes $x=0$, $x=l_{t} / 2$ and $x=l_{t}$. The solution results in:

$$
A_{b_{z h}}(x, y)=\sum_{n=1,3, \ldots}^{\infty}\left(k_{7} e^{\kappa n y}+k_{8} e^{-\kappa n y}\right) \operatorname{sen}(\kappa n x)
$$

The particular solution is equal to:

$$
\frac{\partial^{2} A_{b_{z p}}}{\partial x^{2}}+\frac{\partial^{2} A_{b_{z p}}}{\partial y^{2}}=-\mu_{o} J_{n} \sin (\kappa n x)
$$

where $J_{n}=\frac{4 J_{O}}{\pi n} \cos \left(k n l_{d}\right)$.The simplest solution can be obtained integrating it twice in $x$, resulting in:

$$
A_{b_{z p}}=\mu_{o} \frac{4 J_{o}}{\pi \kappa^{2}} \sum_{n=1,3, \ldots}^{\infty} \frac{1}{n^{3}} \cos \left(\kappa n l_{d}\right) \sin (\kappa n x)
$$

The complete equation of $A_{b_{z}}$ results in:

$$
A_{b_{z}}(x, y)=A_{b_{z h}}+A_{b_{z p}}=\sum_{n=1,3, \ldots}^{\infty}\left[\left(k_{7} e^{\kappa n z}+k_{8} e^{-\kappa n z}\right)+\mu_{o} \frac{4 J_{o}}{\pi \kappa^{2} n^{3}} \cos \left(\kappa n l_{d}\right)\right] \sin (\kappa n x)
$$

In the ferromagnetic armature core, under the plane $y=0$, the magnetic vector potential also assumes the form of the Laplace's equation, according to (24):

$$
A_{f e_{z}}(x, y)=\sum_{n=1,3, . .}^{\infty}\left(k_{5} e^{\kappa n y}+k_{6} e^{-\kappa n y}\right) \sin (\kappa n x)
$$

The region between the boundaries $B$ and $G$, Fig. 2, is free from currents. So, the equation of the magnetic vector potential assumes the form of the Laplace's equation, according to (25):

$$
A_{g b_{z}}(x, y)=\sum_{n=1,23}^{\infty}\left(k_{9} e^{\kappa n y}+k_{10} e^{-\kappa n y}\right) \sin (\kappa n x)
$$


The region between the boundaries $G$ and $P$, Fig. 2, is also free from currents. The equation of the magnetic vector potential also assumes the form of the Laplace's equation and is given by (26).

$$
A_{p m b_{z}}(x, y)=\sum_{n=1,23}^{\infty}\left(k_{11} e^{\kappa n y}+k_{12} e^{-\kappa n y}\right) \sin (\kappa n x)
$$

Equations (23) to (26) have eight unknowns, i.e. $k_{5}, k_{6}, k_{7}, k_{8}, k_{9}, k_{10}, k_{11}$ and $k_{12}$ whose obtaining depends on the conditions of the magnetic field when $y \rightarrow \infty$ and $y \rightarrow-\infty$ and on the boundaries $O, B$ and $G$, Fig. 2. The conditions of the magnetic field are the following [6]:

- when $y \rightarrow-\infty, B_{f e_{x}}=\left(\frac{\partial A_{f e_{z}}}{\partial y}\right)=0$. This condition implies that $k_{6}$ must be equal to zero;

- on boundary $O$, where $y=0$, the $y$-components of the magnetic flux density vector in the ferromagnetic core of the armature and in the winding are equal, $B_{f e_{y}}^{O}=B_{b_{y}}^{O}$, or $\left(\frac{\partial A_{f e_{z}}}{\partial x}\right)_{y=0}=\left(\frac{\partial A_{b z}}{\partial x}\right)_{y=0}$ and the $x$-components of the magnetic field vector in the ferromagnetic core of the armature and in the winding are equal, too, or $H_{f e_{x}}^{O}=H_{b_{x}}^{O}$, or $\frac{1}{\mu_{f e}}\left(\frac{\partial A_{f e_{z}}}{\partial y}\right)_{y=0}=\frac{1}{\mu_{0}}\left(\frac{\partial A_{b_{z}}}{\partial y}\right)_{y=0}$, where $\mu_{f e}$ is the magnetic permeability of the armature core;

- on boundary $B$, where $y=l_{b}$, the $y$-components of the magnetic flux density vector in the windings and in the free space in air are equal, $B{ }_{b_{y}}^{B}=B{ }_{g b_{y}}^{B}$, or $\left(\frac{\partial A_{b_{z}}}{\partial x}\right)_{y=l_{b}}=\left(\frac{\partial A_{g b_{z}}}{\partial x}\right)_{y=l_{b}}$ and the $x$-components of the magnetic field vector in the windings and in the free space in air are equal, too, $H_{b_{x}}^{B}=H_{g b_{x}}^{B}$, or $\left(\frac{\partial A_{b_{z}}}{\partial y}\right)_{y=l_{b}}=\left(\frac{\partial A_{g b_{z}}}{\partial y}\right)_{y=l_{b}}$ since the free space in air and the winding region have the same magnetic permeability. Here, $B_{b_{y}}$ and $B_{g b_{y}}$ correspond to the y-components of the magnetic flux density vector in the region of windings and in the free space in air, respectively, ${ }_{b x}$ and ${ }_{g b_{x}}$ correspond to the x-components of the magnetic field vector in the region of windings and in the free space in air, respectively. The superscript $B$ denotes the boundary $B$;

- on boundary $G$, Fig. 2, where $y=l_{g}$, the $y$-components of the magnetic flux density vector 
in the free space in air and in the permanent magnets region are equal, $B_{g_{b y}}^{G}=B{ }_{p m b_{y}}^{G}$, or $\left(\frac{\partial A_{g b_{z}}}{\partial x}\right)_{y=l_{g}}=\left(\frac{\partial A_{p m b_{z}}}{\partial x}\right)_{y=l_{g}}$ and the $x$-components of the magnetic field vector in the free space in air and in the permanent magnets region are equal, too, $H_{g b_{x}}^{G}=H_{p m b_{x}}^{G}$, or $\frac{1}{\mu_{0}}\left(\frac{\partial A_{g b_{z}}}{\partial y}\right)_{y=l_{g}}=\frac{1}{\mu_{p m}}\left(\frac{\partial A_{p m b} z}{\partial y}\right)_{y=l_{g}}$. Here, $\mu_{p m}$ is the magnetic permeability of the permanent magnets, $B_{p m b}$ corresponds to the y-component of the magnetic flux density vector in the region of the permanent magnets and ${ }_{p m b}$ corresponds to the x-component of the magnetic field vector in the region of the permanent magnets. The superscript $G$ denotes boundary $G$, Fig. 2;

- $\quad$ when $y \rightarrow \infty, B_{p m b_{x}}=0$. This condition implies that $k_{11}$ must be equal to zero.

The resulting equations of the magnetic vector potential in the winding region and in the free space in air are given, respectively, by:

$$
A_{b_{z}}(x, y)=\sum_{n=1,3, \ldots}^{\infty} \frac{A_{p}}{2}\left[\left[\left(\left(\frac{\mu_{r_{f e}}+1}{\mu_{r_{f e}}-1}\right) M_{n}+2\right) e^{k n y}+M_{n} e^{-\kappa n y}+2\right]\right] \sin \left(\kappa n\left(x-\frac{l_{t}}{8}\right)\right)
$$

and

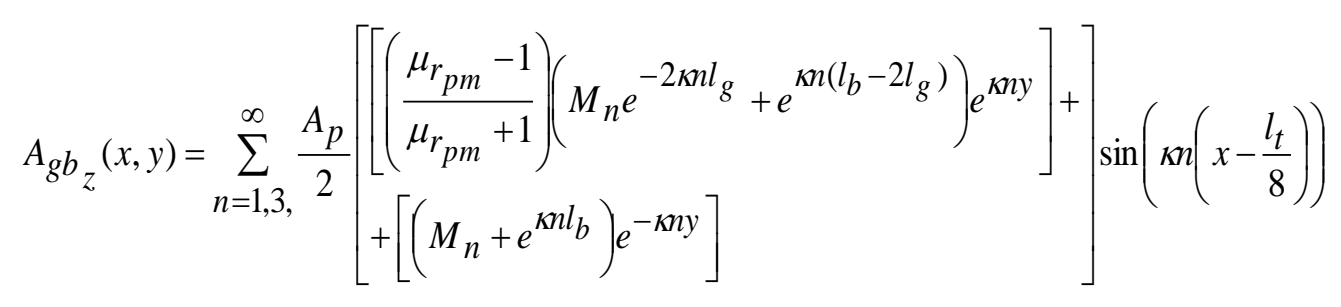

where $A_{p}=\mu_{o} \frac{4 J_{o}}{\pi \kappa^{2}} \frac{1}{n^{3}} \cos \left(\kappa n l_{d}\right)$ and $\mu_{r_{f e}}$ is the relative magnetic permeability of the ferromagnetic core of the armature and $M_{n}$ is given by (29). 


$$
M_{n}=\left[\frac{\left(\mu_{r_{f e}}-1\right)\left(\mu_{r_{p m}}-1\right) e^{2 \kappa n\left(l_{b}-l_{g}\right)}-2\left(\mu_{r_{p m}}+1\right) e^{k n l_{b}}-\left(\mu_{r_{f e}}-1\right)\left(\mu_{r_{p m}}+1\right)}{\left(\mu_{r_{f e}}+1\right)\left(\mu_{r_{p m}}+1\right) e^{k n l_{b}}-\left(\mu_{r_{f e}}-1\right)\left(\mu_{r_{p m}}-1\right) e^{\kappa n\left(l_{b}-2 l_{g}\right)}}\right]
$$

The $x$ and $y$-components of the magnetic flux density vector in the free space in air, $B_{g b_{x}}(x, y)$ and $B_{g b_{y}}(x, y)$ are generate by $J_{z}$. These two quantities are obtained, respectively, by $\frac{\partial A_{g b}}{\partial y}$ and $-\frac{\partial A_{g b}}{\partial x}$. Their expressions are presented in (30) and (31).

$$
\begin{aligned}
& B_{g b_{x}}(x, y)=\sum_{n=1,3,}^{\infty} \kappa \frac{A_{p}}{2}\left[\begin{array}{l}
\left.\left[\left(\frac{\mu_{r_{p m}}-1}{\mu_{r_{p m}}+1}\right)\left(M_{n} e^{-2 \kappa n l_{g}}+e^{\kappa n\left(l_{b}-2 l_{g}\right)}\right) e^{\kappa n y}\right]+\right] \sin \left(\kappa n\left(x-\frac{l_{t}}{8}\right)\right) \\
\left.-\left[\left(M_{n}+e^{\kappa n l}\right)\right) e^{-\kappa n y}\right]
\end{array}\right. \\
& B_{g b_{y}}(x, y)=\sum_{n=1,3,}^{\infty}(-\kappa n) \frac{A_{p}}{2}\left[\begin{array}{l}
\left.\left[\left(\frac{\mu_{r_{p m}}-1}{\mu_{r_{p m}}+1}\right)\left(M_{n} e^{-2 \kappa n l_{g}}+e^{\kappa n\left(l_{b}-2 l_{g}\right)}\right) e^{\kappa n y}\right]+\right] \cos \left(\kappa n\left(x-\frac{l_{t}}{8}\right)\right) \\
+\left[\left(M_{n}+e^{\kappa n l_{b}}\right) e^{-\kappa n y}\right]
\end{array}\right]
\end{aligned}
$$

The magnetic flux density in the air gap depends on the fields produced by currents in the armature winding and by the permanent magnets. Between the boundaries $O$ and $B$, Fig. 2, located between the planes $y=0$ and $y=l_{b}$, the equation of the $x$ and $y$-components of the magnetic flux density vector is equal to $B_{T_{x}}{ }^{O B}=B_{g_{x}}(x, y)+B_{b_{x}}(x, y)$ and $B_{T_{y}}{ }^{O B}=B_{g_{y}}(x, y)+B_{b_{y}}(x, y)$. Between the boundaries $B$ and $G$, Fig. 2, the same components are equal to $B_{T_{x}}{ }^{B G}=B_{g_{x}}(x, y)+B_{g b_{x}}(x, y)$ and $B_{T_{y}}{ }^{B G}=B_{g_{y}}(x, y)+B_{g b_{y}}(x, y)$. The subscript $T$ denotes the total value of the components of the magnetic flux density vector.

\section{The Linear Propulsion Force}

The linear propulsion force is deduced by means of Laplace's force equation, $\vec{F}=\int_{V}(\vec{J} \times \vec{B}) d V$. In this equation, the force results of the volume integral of the vector product between the density current vector and the magnetic flux density vector through the active volume of the conductors fed by current. The propulsion force that acts over the mover can be deduced by the force that acts over the permanent magnet, according (32). The negative vector product between the $y$-component of the magnetic flux density in the air gap produced by the permanent magnets and the density current 
vector in the armature winding, integrated under the active volume of the phases located under the permanent magnets with normal magnetization produces a force vector with a $x$-component equal to the propulsion force that produces the linear displacement of the mover through the $x$-axis. The result of (33) is presented in (34), where $l_{p}$ is active length of the coils through $z$-axis.

$$
\begin{aligned}
& \vec{F}_{P}=4 \int_{V}-\left(J_{z} \vec{k} \times B_{g_{y}} \vec{j}\right) d V=4 \int_{V}\left(B_{g_{y}} \vec{j} \times J_{z} \vec{k}\right) d V=4 \int_{V}\left(B_{g_{y}} J_{z}\right) \vec{i} d V \\
& \vec{F}_{P}=\sum_{n=1,3, . .}^{\infty} \frac{16 \kappa n l_{p} \mu_{O} J_{o}}{\pi n} \cos \left(\kappa n l_{d}\right) \int_{0}^{l_{b}} \int_{\frac{l_{t}}{4}}^{\frac{l_{t}}{2}}\left[\left(\frac{\left(e^{\kappa n y}+e^{-\kappa n y}\right)}{a}\right)\left(b \psi_{p}+c \frac{M_{y}}{\kappa n}\right) \sin \left(\kappa n\left(x-\frac{l_{t}}{8}\right)\right)\right] \vec{i} d x d y \\
& \vec{F}_{P}=\frac{16 l_{p} \mu_{O} J_{O} M_{O}}{\kappa \pi^{2}} \sum_{n=1,3, \ldots}^{\infty} \frac{\cos \left(\kappa n l_{d}\right)}{n^{3}}\left(\frac{\left(e^{\left.k n l_{b}-e^{-\kappa n l_{b}}\right)}\right.}{a}\right)\left[\begin{array}{l}
{\left[\left(b b_{3}+c b_{2}\right)\left(-\frac{1}{2 \kappa n} \cos \left(\kappa n \frac{l t}{8}\right)-\frac{l t}{8} \sin \left(\kappa n \frac{l t}{8}\right)\right)\right]+} \\
+\left[\left(b b_{2}+c b 3\right)\left(\frac{l t}{8} \cos \left(\kappa n \frac{l t}{8}\right)+\frac{1}{2 \kappa n} \sin \left(\kappa n \frac{l t}{8}\right)\right)\right]
\end{array}\right] \vec{i}
\end{aligned}
$$

where

$$
\begin{gathered}
a=2\left(e^{k n l_{g}}-e^{-\kappa n l_{g}}\right)\left(e^{2 \kappa n\left(l_{m}+l_{g}\right)}-1\right) \\
b=\left(\left(e^{\kappa n l_{g}}-e^{\kappa n\left(2 l_{m}+l_{g}\right)}\right)\left(e^{\kappa n l_{g}}+e^{-\kappa n l_{g}}-2 e^{\kappa n\left(l_{m}+l_{g}\right)}\right)+2\left(e^{2 \kappa n\left(l_{m}+l_{g}\right)}-1\right)\left(1-e^{\kappa n l_{m}}\right)\right) \\
b_{2}=\sin \frac{\pi n}{2}-\sin \frac{3 \pi n}{2} \\
b_{3}=\cos (\pi n)-1 \\
c=\left(e^{\kappa n l_{g}}-e^{-k n l_{g}}\right)\left(e^{\kappa n l_{g}}-e^{\kappa n\left(2 l_{m}+l_{g}\right)}\right)
\end{gathered}
$$

\section{The Normal Force}

A normal force is also present, and it is the result of the magnetic attraction between the permanent magnets and ferromagnetic core of the armature. The propulsion force was obtained by Laplace's force. In the analysis of the normal force, Maxwell Tensor was employed for obtaining its equation.

The force vector, $\vec{F}$, is related to the Maxwell tensor by $\vec{F}=\frac{1}{\mu} \oint_{S} \vec{T} d S$, where $\vec{T}$ is Maxwell tensor [4]. The differential of force that acts through $y$-axis, $d F_{y}$, in $\mathrm{Nm}^{-2}$, is calculated by 
$d F_{y}=\frac{1}{\mu}\left(T_{y x} d y d z+T_{y y} d x d z+T_{y z} d x d y\right)$, where $T_{y x}$ is equal to $B_{x} B_{y}, T_{y y}$ to $B_{y}^{2}-\frac{1}{2}|\vec{B}|^{2}$ and $T_{y z}$ to $B_{z} B_{y}$ [10]. The integration of $d F_{y}$ over a closed surface that involves entirely the permanent magnets produces the normal force the acts between the permanent magnets and the ferromagnetic material of the armature. The lower surface of the closed surface is located on the boundary $G$. The upper surface is located on $y=+\infty$ where the magnetic field is considered equal to zero, so, the integration over this surface is zero. On the laterals sides, the integration over the terms $T_{y x}$ and $T_{y z}$ cancel one to each other [2]. By this way, the normal force results of the integration of $T_{y y}$ through the surface located on boundary $G\left(y=l_{g}\right)$, where are located lower polar surfaces of the permanent magnets, according (40).

$$
\vec{F}_{y}=\vec{F}_{N}=\frac{1}{\mu_{o}} \iint T_{y y} \vec{j} d x d z=\frac{1}{\mu_{o}} \iint\left(B_{y}{ }^{2}-0.5|\vec{B}|^{2}\right) \vec{j} d x d z=\frac{1}{2 \mu_{o}} \iint\left(B_{y}{ }^{2}-B_{x}{ }^{2}\right) \vec{j} d x d z
$$

The $x$ and $y$-components of (40) result of the contribution of the magnetic field produced by the current in the armature phases and of the magnetic field produced by the Halbach array. In the present analysis, the magnetic field is consider constant through the $z$-axis, so (40) results in (41), where $l_{p}$ is active length of the coils through $z$-axis. The upper limit of integration, $L_{t}$, corresponds to the entire length of the Halbach array through $x$-axis and it is obtained by the sum of $2 l_{t}$ and $\frac{l_{t}}{4}$.

$$
F_{N}=\frac{l_{p}}{2 \mu_{o}} \int_{0}^{L_{t}}\left\langle\left(\left(B_{T_{y}} G\right)^{2}-\left(B_{T_{x}} G\right)^{2}\right)\right\rangle_{x} \vec{j} d x
$$

In (41), the symbol \langle\rangle$_{x}$ indicates the mean value of the operation over $x$. The result of (41) is given by (42):

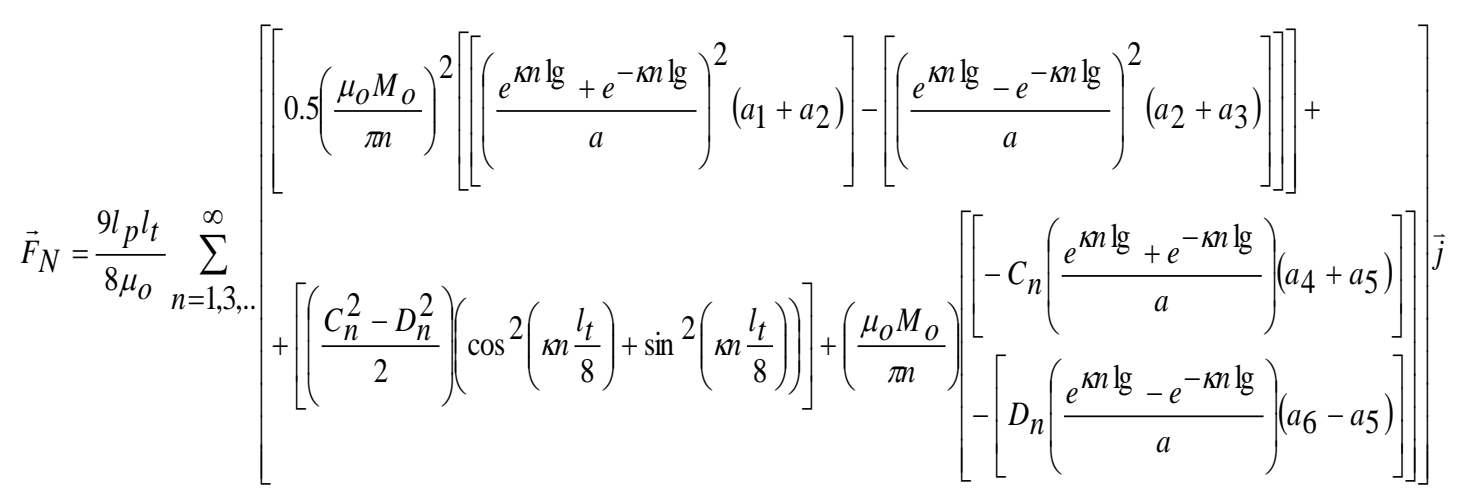

where:

$$
a_{1}=\left(\left(b b_{3}\right)+\left(c b_{2}\right)\right)^{2}
$$




$$
\begin{aligned}
& a_{2}=\left(\left(b b_{2}\right)+\left(c b_{3}\right)\right)^{2} \\
& a_{3}=\left(\left(b b_{1}\right)+\left(c b_{4}\right)\right)^{2} \\
& a_{4}=\left(\left(b b_{3}\right)+\left(c b_{2}\right)\right) \cos \left(\kappa n \frac{l_{t}}{8}\right) \\
& a_{5}=\left(\left(b b_{2}\right)+\left(c b_{3}\right)\right) \sin \left(\kappa n \frac{l_{t}}{8}\right) \\
& a_{6}=\left(\left(b b_{1}\right)+\left(c b_{4}\right)\right) \cos \left(\kappa n \frac{l_{t}}{8}\right) \\
& b_{1}=1-\cos (\pi n) \\
& b_{4}=\sin \frac{3 \pi n}{2}-\sin \frac{\pi n}{2} \\
& C_{n}=\kappa n \frac{A_{p}}{2}\left[\left[\left(\frac{\mu_{r_{p m}}-1}{\mu_{r_{p m}}+1}\right)\left(M_{n} e^{-\kappa n l_{g}}+e^{\kappa n\left(l_{b}-l_{g}\right)}\right)\right]+\left[\left(M_{n}+e^{\kappa n\left(l_{b}-l_{g}\right)}\right)\right]\right] \\
& D_{n}=\kappa n \frac{A_{p}}{2}\left[\left[\left(\frac{\mu_{r_{p m}}-1}{\mu_{r_{p m}}+1}\right)\left(M_{n} e^{-\kappa n l_{g}}+e^{\kappa n\left(l_{b}-l_{g}\right)}\right)\right]-\left[\left(M_{n}+e^{\kappa n\left(l_{b}-l_{g}\right)}\right)\right]\right]
\end{aligned}
$$

In order to validate the analytical model, the linear motor was modelled using FEMM 4.2. A numerical model was constructed with the same characteristics of those showed in Table I. To take into account the effects of magnetic flux lines that pass through the air, the motor was encircled in all directions by a region of air. The simulations of the motor were carried under static conditions. In order to compare the results of the analytical model, the linear motor was numerically analysed without current in its armature phases. The results gave an assessment of the distribution of the magnetic flux in the air gap produced by the Halbach array. After, the linear motor was analysed with current in the coils, in order to verify the effect of the magnetic field produced by current in the armature winding on the normal force.

\section{E. Measurements}

A prototype of the linear motor presented in Fig. 1 was constructed and it helped to validate the analytical model by means of measurement of propulsion and normal forces. A test rig was designed for the experimental part of the study. Measurements of forces under static conditions were taken 
using force sensing resistors. It provides an inverse change in resistance in response to an increase/decrease in applied force. Fig. 5(a) presents the test rig for measurement of propulsion force with the actuator suspended by means of a structure that keeps it static. During the tests, only four phases located under permanent magnets with normal magnetization were fed by current. Fig. 5(b) shows the force sensing resistor and its position in the structure used for measurements.

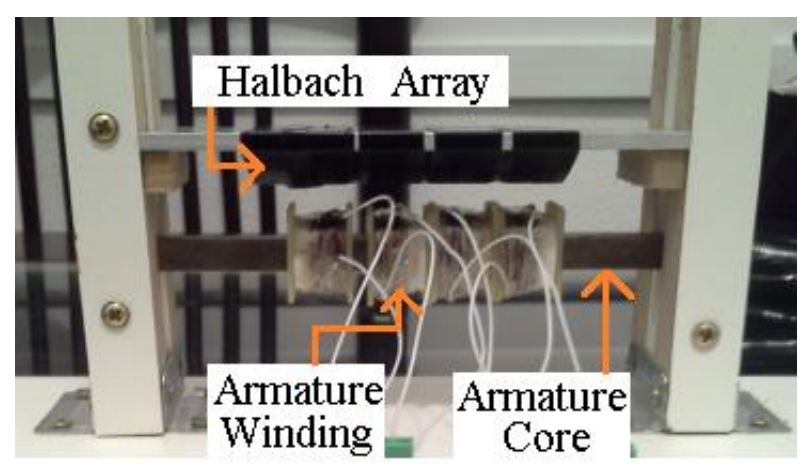

(a)

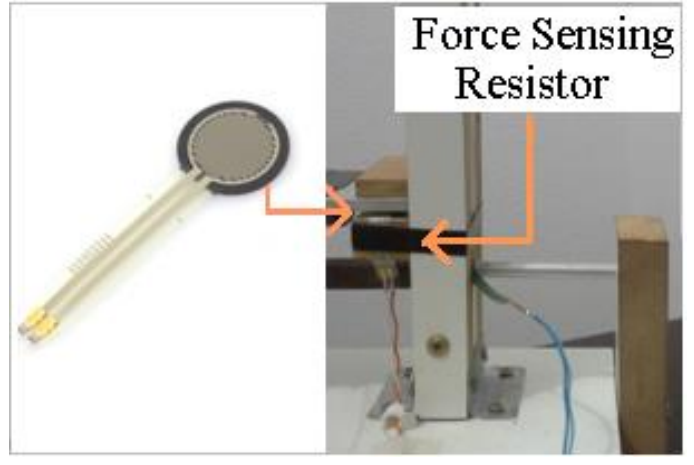

(b)

Fig. 5. a) Test rig for measurement of force; b) force sensing resistor and its position in the structure used for measurements.

\section{RESULTS AND DISCUSSION}

Fig. 6 presents the graph of the $y$-component of magnetic flux density vector in the air gap vs. position on the plane underneath the mover at $y=6 \mathrm{~mm}$, when current in the phases of the armature is zero. The graph presents values obtained by the analytical method described in this paper, using (16). For comparison and validation of the analytical model, curves are obtained by numerical analysis and by measurements, under the same conditions of current and position used in the analytical method. The measured values were obtained by means of a gaussmeter. The behaviour of the magnetic flux density in the plane $\mathrm{y}=6 \mathrm{~mm}$ presents a sinusoidal shape as expected instead a flattened shape.

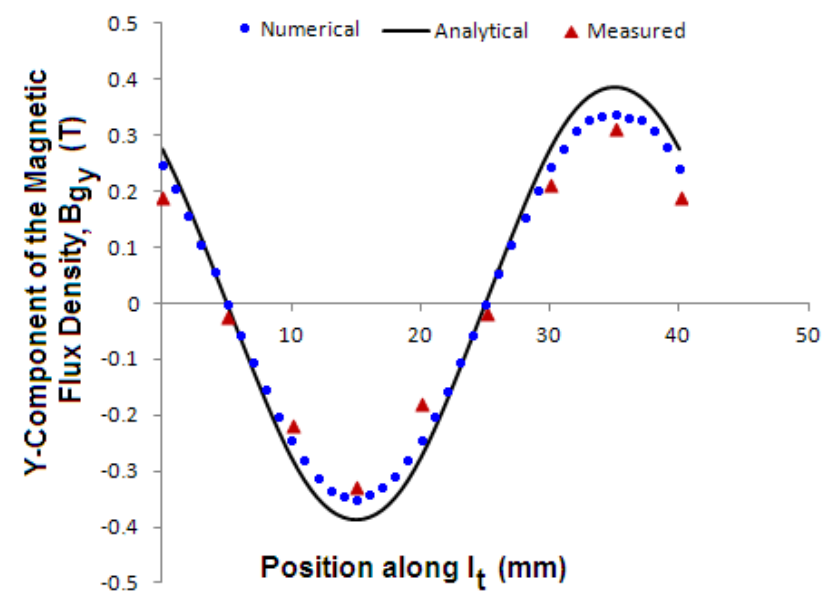

Fig. 6. Graph of the $y$-component of the magnetic flux density vector in the plane $y=6 \mathrm{~mm}$, with current equal to zero.

The numerical analysis allowed to foresee the behaviour of the distribution of the magnetic flux density in the structure of the linear motor, especially in the ferromagnetic core of the armature, considered infinitely permeable in the analytical model. The results of the numerical simulations 
allowed verifying that the core did not present effect of saturation and, in that region, the maximum value of the modulus of the magnetic flux density vector is $0.3 \mathrm{~T}$. Fig. 7(a) presents the behaviour of the magnetic flux density in the magnetic circuit of the linear motor produced by the Halbach array obtained by numerical analysis with current in the armature winding equal to zero. Fig. 7(b) and 7(c) presents the respective graphs of the $y$-component of magnetic flux density vector in the air gap vs. position on the plane underneath the mover at $y=10 \mathrm{~mm}$ and $y=0$. Analytical results are presented, too. In the planes near the polar surfaces of the Halbach array, the behaviour of the distribution of the magnetic flux density presents a flattened aspect, Fig. 7(b). According to the expected, in the region of the coils, the distribution of the $y$-component of the magnetic flux density vector presented a shape very closed to a sinusoidal behaviour, Fig. 7(c). The coils are located under the plane $y=6 \mathrm{~mm}$, where the distribution of the magnetic flux density has a quasi-sinusoidal behaviour. This characteristic results in a propulsion force with no significant odd harmonics, according to the analytical model. The fundamental value of the propulsion force is $2.03 \mathrm{~N}$, and the $3^{\text {th }}$ harmonic, $5.74 \times 10^{-5} \mathrm{~N}$, with a current of $1.45 \mathrm{~A}$.

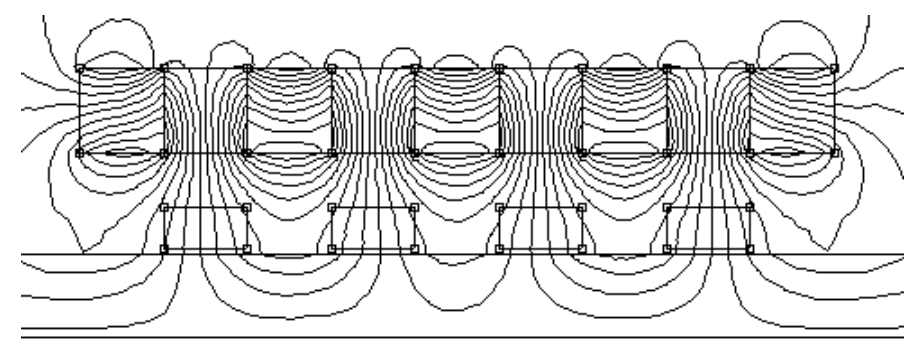

(a)

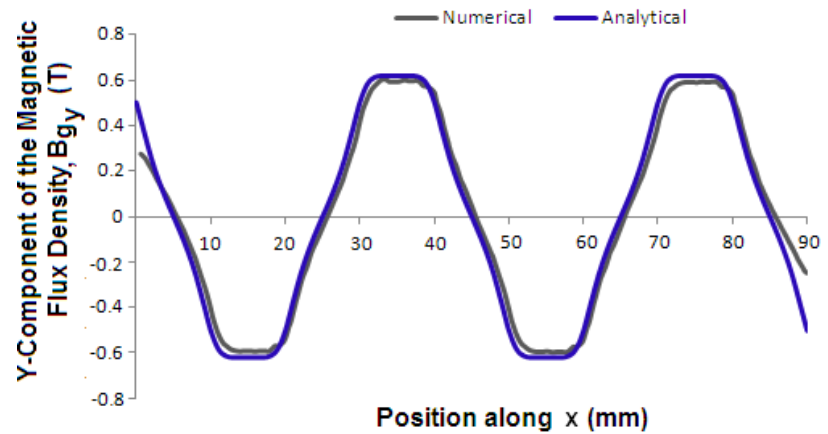

(b)

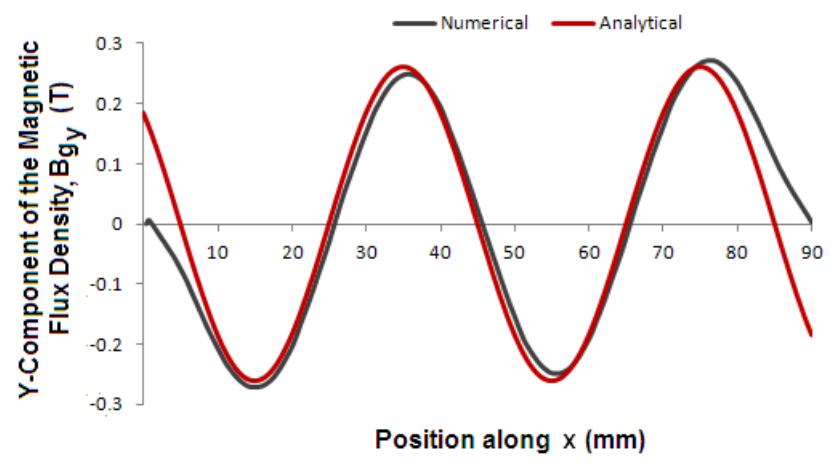

(c)

Fig.7. (a) Shape of the magnetic flux distribution produced by the Halbach array in the linear motor obtained by numerical analysis; (b) and (c) graphs of the $y$-component of magnetic flux density vector in the air gap at $\mathrm{y}=10 \mathrm{~mm}$ and $\mathrm{y}=0$. 
Fig. 8 shows the graph of the measured linear propulsion force on static conditions, when only the phases located under the permanent magnets with normal magnetization were fed by current. By means of the values presented in the graph of propulsion force, the mean sensitivity in N/A was calculated. Table II presents the resulting mean sensitivities obtained for measurements and theoretical values of propulsion force. Taking into account the measured results as a reference, the difference between the measured and the numerical values is $1.47 \%$, and between the measured and analytical ones, $2.94 \%$.

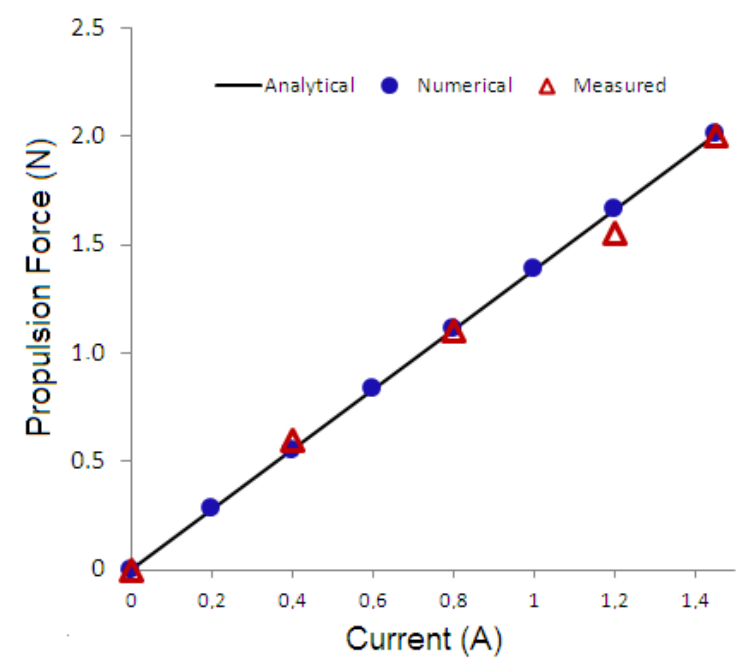

Fig. 8. Graph of the propulsion force vs. current in the coils of the armature winding.

TABLE II. MEAN SENSITIVITY OF THE LINEAR MOTOR UNDER STUDY.

\begin{tabular}{ccc}
\hline & $\begin{array}{c}\text { Average Sensitivity } \\
\text { (N/A) }\end{array}$ & $\begin{array}{c}\text { Difference } \\
(\boldsymbol{\%})\end{array}$ \\
\hline Analytical Method & 1.40 & +2.94 \\
Numerical Analysis & 1.38 & +1.47 \\
Measured & 1.36 & - \\
\hline
\end{tabular}

Fig. 9 presents the graph of the normal force obtained with the same conditions imposes for obtaining the propulsion force. For comparison and validation, figures were obtained by the analytical model and by the numerical analysis under the same values of current of those obtained by the measurements. The analytical values of propulsion force were calculated using (34), and the normal force was obtained by (42).

The present study also helped to analyse the behaviour of propulsion and normal forces as a function of the air gap length. Fig 10 shows the graph of the propulsion force as a function of the air gap length, when current in the coil located under the permanent magnets with normal magnetization is 1.45 A. Fig. 11 shows the graph of the normal force vs. the air gap length obtained with the same conditions of the graph of the propulsion force. The normal force is the principal characteristic employed to design the suspension system of the linear motor, since it must compensate the effect of a large normal force. 


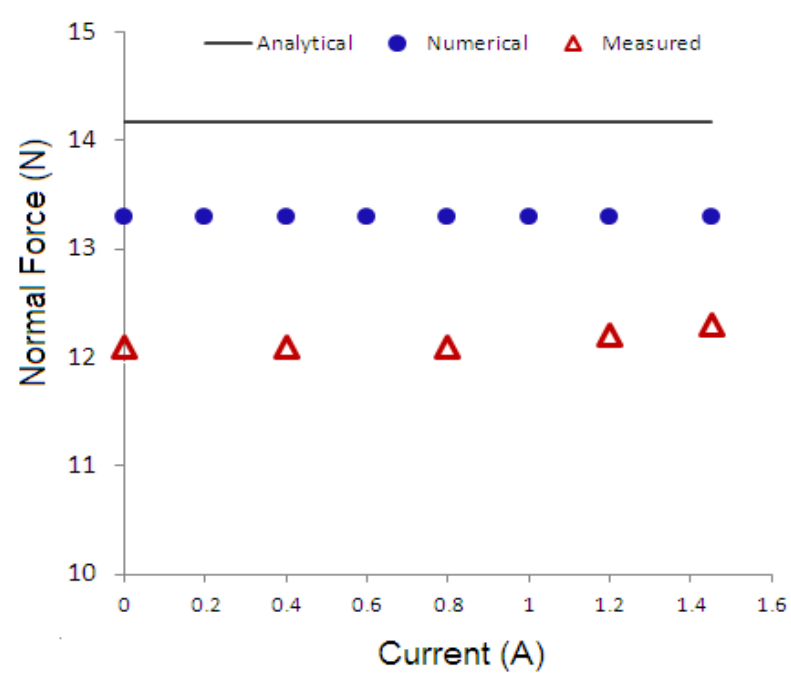

Fig.9. Graph of the normal force vs. current in the coils of the armature winding.

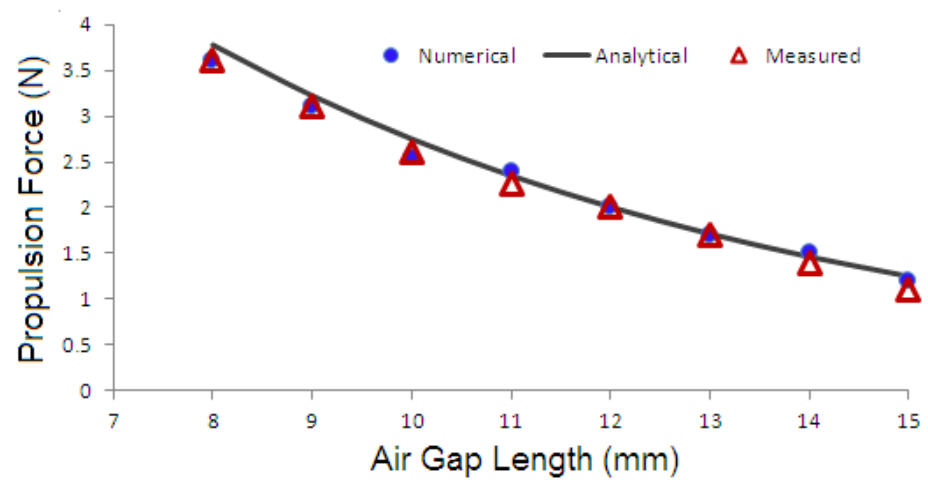

Fig. 10. Graph of the propulsion force vs. air gap length.

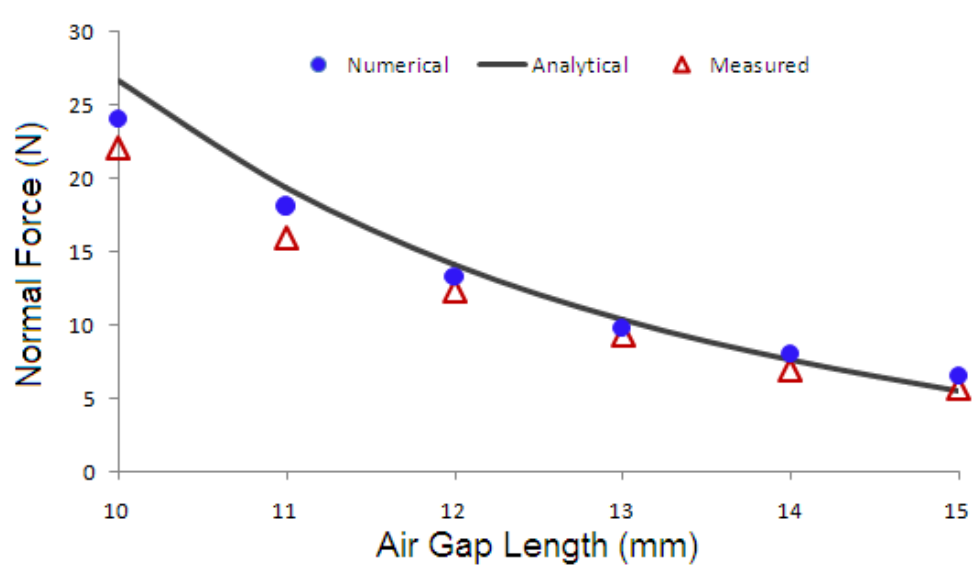

Fig. 11. Graph of the normal force vs. air gap length.

\section{CONCLUSIONS}

The theoretical models show good computation of the produced forces, and will help in further analysis. One of the goals of the design of the linear motor was to guarantee that the coils of the armature winding were immersed in a distribution of magnetic flux density vector produced by the permanent magnets with a sinusoidal behaviour. The theoretical result allowed verifying that between 
the planes $y=0$ and $y=6 \mathrm{~mm}$ the $y$-component of the magnetic flux density had a very near sinusoidal shape. The involved forces have odd harmonics of very small values and, in the calculation by analytical method, the results converge quickly.

The magnetic field produced by the current in the armature winding are very small when compared to the magnetic field produced by the permanent magnets of the Halbach array. The effect of the magnetic field produced by currents on the normal force is not significant, according theoretical results. Considering a variation from 0 to the rated value of current $(1.45 \mathrm{~A})$, the theoretical normal force did not present modification in its values. Experimental results presented a slight influence of the armature current on the normal force. When current is equal to zero, the measured value of normal force is $12.1 \mathrm{~N}$ and, with a current of $1.45 \mathrm{~A}$, is $12.5 \mathrm{~N}$.

The analysis of the results of the $y$-component of the magnetic flux density vector, Fig. 6, allows one to conclude that the difference between the simulated and measured peak values is $5.98 \%$ and between the analytical and measures peak values is $16.95 \%$ in the plane $y=6 \mathrm{~mm}$. In the same plane, the analytical average value of the $y$-component of the magnetic flux density vector is 0.234 $\mathrm{T}$, while the numerical one is $0.209 \mathrm{~T}$. Those percentage values vary according to the $y$-coordinate. As one can see in Fig. 7(b), in $y=10 \mathrm{~mm}$, near the polar surface of the permanent magnets with normal magnetization, the analytical and numerical values of the $y$-component of the magnetic flux density vector have a smaller difference.

With relation to the propulsion force, in the sensitivity comparison, the average difference between the simulated and the measured values is $1.47 \%$ and between analytical and measured values, $2.94 \%$. The normal force difference is $8.93 \%$ between the simulated and the measured values, and $16.78 \%$ between analytical and measured values, Fig. 9. Propulsion and normal forces presented a nonlinear behaviour with respect of the variation of the air gap length. The propulsion force difference between the simulated and measured values is $1.85 \%$, and between the analytical and measured values is $4.46 \%$, Fig. 10. In the case of normal force, between the simulated and measured values, the difference is $10.51 \%$, and between the analytical and measured values is $15.72 \%$, Fig. 11 .

In the propulsion force calculation, it was employed the Lorentz-Laplace force. The force was obtained by the vector product of the $y$-component of the magnetic flux density vector and the $\mathrm{z}$ component of the current density vector integrated under the active volume of the coils under current. In the region of the coils, the difference between the analytical and numerical values of the $y$-component of the magnetic flux density vector varies from $6.9 \%$ to $12.05 \%$. The differences in the propulsion force sensitivity comparison are much smaller. It was expected a larger difference between the results of force due to the differences in the comparison of the $y$-component of the magnetic flux density vector. Possibly, that result was due to errors introduced by the correction factor applied to the density current calculation in order to obtain the effective crosssectional area occupied by the conductors in the winding region. The analytical results of 
propulsion force was obtained by integration over $l_{b}$ and $l_{t} / 4$ (the cross-sectional area of one coil is $\left.s_{c}=l_{b}\left(l_{t} / 4\right)\right)$. The conductors occupy an effective cross-sectional area smaller than $s_{c}$.

Analytical models based on Fourier series can be a good tool for understanding and previewing the behaviour of the magnetic flux density in electromagnetic devices. The theoretical models show good computation of the produced forces, and will help in further analysis. The analytical model allows understanding the behaviour of the magnetic flux density in the air gap and in the components of the linear motor and calculating the forces.

The equations of the analytical model lead to the fast computation of the fields and forces. The numerical analysis allows the verification of the conditions of the magnetic field in the ferromagnetic material of the motor. The concomitant use of those two theoretical models and their results helps on the design of the linear motors with permanent magnets and on the analysis of the impact on the values of forces when modifications on dimensions are made. From the education point of view, students can benefit from this work in many ways: to learn the electromagnetic modelling approach by means of fundamental equations; by applying that approach to a special topology of linear motor; and by seeking validation of the study by means of numerical e test results.

\section{ACKNOWLEDGMENT}

The authors would like to acknowledge the financial support that allowed the development of this work provided by the National Council for Scientific and Technological Development (CNPq) of Brazil.

\section{REFERENCES}

[1] Jean Baptiste Joseph Fourier, Théorie Analytique de la Chaleur. Paris: F. Didot, 1822.

[2] D. L. Trumper, M. E. Williams, T. H. Nguyen, "Magnet arrays for synchronous machines," Proc. IEEE IAS 28th Annu. Meet., pp. 9-18, 1993.

[3] K. J.Meessen,, B. L. J. Gysen,; J. J. H. Paulides, H.; E. A. Lomonova, 'Halbach permanent magnet shape selection for slotless tubular actuators,' IEEE Transaction on Magnetics, vol. 44, n. 11, pp. ,Nov. 2008.

[4] J. R. Melcher, Continuum Electromechanics. Cambridge, MS: MIT Press, 1981.

[5] A. F.Flores Filho, A. A.Susin, M. A. da Silveira, "An Analytical Method to Predict the Static Performance of a Planar Actuator,” IEEE Transaction on Magnets, vol. 39, pp. 3364-3366, Sep 2003.

[6] K. J. Binns, P. J. Lawrenson and C. W. Trowbridge, Electric and Magnetic Fields. West Sussex: John Wiley \& Sons, 1992.

[7] W. J. Kim, High-Precision Planar Magnetic Levitation. Ph.D. Thesis, Massachusetts Institute of Techonology, Cambridge, MS, 1997.

[8] M. G. Lee, S. Q Lee, D.-G. Gweon, "Analysis of Halbach magnet array and its application to linear motor," Mechatronics, vol. 10, pp. 115-128, Feb 2004.

[9] H. Allag, J-P. Yonnet, M. Latreche, "3D analytical calculation of forces between linear Halbach-type permanentmagnet arrays", Proc. 8th Int. Symp. Adv. Electromech. Motion Syst. Electric Drives Joint Symp. (ELECTROMOTION), pp. 1-6, 2009.

[10] . F.Flores Filho, M. A. da Silveira, R. P. Homrich, "Evaluation of the normal force of a planar actuator", IEEE Transactions on Magnetics, v. 41, pp. 4006-4008, Oct 2005

Brazilian Microwave and Optoelectronics Society-SBMO received 30 Oct 2012; for review 03 Nov 2016; accepted 28 Dec 2016 Brazilian Society of Electromagnetism-SBMag 